\title{
Euler Type Half-Linear Differential Equation with Periodic Coefficients
}

\author{
Ondřej Došlý and Hana Funková \\ Department of Mathematics and Statistics, Masaryk University, Kotlářská 2, 61137 Brno, Czech Republic \\ Correspondence should be addressed to Ondřej Došlý; dosly@math.muni.cz
}

Received 10 July 2013; Accepted 23 September 2013

Academic Editor: Abdelaziz Rhandi

Copyright (C) 2013 O. Došlý and H. Funková. This is an open access article distributed under the Creative Commons Attribution License, which permits unrestricted use, distribution, and reproduction in any medium, provided the original work is properly cited.

We investigate oscillatory properties of the perturbed half-linear Euler differential equation. We show that the results of the recent paper by O. Došlý and H. Funková (2012) remain to hold when constants in perturbation terms are replaced by periodic functions.

\section{Introduction}

We investigate the influence of perturbations of the Euler half-linear second-order differential equation:

$$
\begin{gathered}
\left(\Phi\left(x^{\prime}(t)\right)\right)^{\prime}+\frac{\gamma_{p}}{t^{p}} \Phi(x(t))=0 \\
\Phi(x):=|x|^{p-2} x, \quad p>1, t \in(0, \infty),
\end{gathered}
$$

with the so-called critical oscillation constant $\gamma_{p}:=$ $((p-1) / p)^{p-1}$ on oscillatory behavior of this equation. Recall that the constant $\gamma_{p}$ is called critical since (1) with $\gamma$ instead of $\gamma_{p}$ is oscillatory if and only if $\gamma>\gamma_{p}$; see [1]. Hence, (1) is a typical example of the so-called conditionally oscillatory half-linear differential equation.

Equation (1) is a special case of the general half-linear differential equation (sometimes called differential equation with the one-dimensional $p$-Laplacian):

$$
\left(r(t) \Phi\left(x^{\prime}\right)\right)^{\prime}+c(t) \Phi(x)=0
$$

with the continuous functions $r>0$ and $c$. It is wellknown, see [2], that the linear oscillation theory (the classical Sturm-Liouville second-order linear differential equation is the special case $p=2$ in (2)) extends almost verbatim to (2). In particular, the equation

$$
\left(r(t) \Phi\left(x^{\prime}\right)\right)^{\prime}+\lambda c(t) \Phi(x)=0
$$

is said to be conditionally oscillatory if there exists $\lambda_{0}>0$ such that (3) is oscillatory for $\lambda>\lambda_{0}$ and nonoscillatory for $\lambda<$ $\lambda_{0}$; see [3]. From this point of view, (1) can be regarded as a good comparative equation in the sense that if $r(t) \equiv 1$ in (2), the first natural test of (non)oscillation of this equation is to compare the potential $c$ with the function $\gamma_{p} t^{-p}$. More precisely, (2) with $r(t) \equiv 1$ is oscillatory provided that

$$
\liminf _{t \rightarrow \infty} t^{p} c(t)>\gamma_{p}
$$

and nonoscillatory if

$$
\limsup _{t \rightarrow \infty} t^{p} c(t)<\gamma_{p}
$$

see, for example, [4, Section 1.4.3].

A natural question is what happens when $\lim _{t \rightarrow \infty} t^{p} c(t)$ $=\gamma_{p}$. This led recently to the investigation of various perturbations of (1). As a first step, the attention was focused on the half-linear Riemann-Weber differential equation and its perturbations:

$$
\left(\Phi\left(x^{\prime}\right)\right)^{\prime}+\left[\frac{\gamma_{p}}{t^{p}}+\sum_{j=1}^{n} \frac{\beta_{j}}{t^{p} \log _{j}^{2}(t)}\right] \Phi(x)=0 .
$$

Here, the notation

$$
\begin{gathered}
\log _{k}(t)=\prod_{j=1}^{k} \log _{k} t, \quad \log _{k} t=\log _{k-1}(\log t), \\
\log _{1} t=\log t
\end{gathered}
$$


is used. It was shown in [5] (see also [6]) that the crucial role in (6) is played by the constant $\mu_{p}:=(1 / 2)((p-1) / p)^{p-1}$. In particular, if $n=1$ in (6), that is, this equation reduces to the so-called Riemann-Weber half-linear differential equation, then this equation is oscillatory if $\beta_{1}>\mu_{p}$ and nonoscillatory in the opposite case. In general, if $\beta_{j}=\mu_{p}$ for $j=1, \ldots, n-1$, then (6) is oscillatory if and only if $\beta_{n}>\mu_{p}$.

One of the typical problems in the qualitative theory of various differential equations is to study what happens when constants in an equation are replaced by periodic functions. Our investigation follows this line and it is mainly motivated by the papers [7-15]. In $[14,15]$, linear second-order differential equations with periodic coefficients were considered which with using a transformation of dependent variable can be transformed into the equation of the following form:

$$
\left(r(t) x^{\prime}\right)^{\prime}+\frac{1}{t^{2}}\left[c(t)+\frac{d(t)}{\log ^{2} t}\right] x=0,
$$

with $\alpha$-periodic functions $r, c, d$. It was shown that (8) behaves essentially in the same way as the classical RiemannWeber equation where the functions $r^{-1}, c, d$ are replaced by their mean values

$$
\begin{gathered}
\bar{r}=\frac{1}{\alpha} \int_{0}^{\alpha} r^{-1}(t) d t, \quad \bar{c}=\frac{1}{\alpha} \int_{0}^{\alpha} c(t) d t \\
\bar{d}=\frac{1}{\alpha} \int_{0}^{\alpha} d(t) d t .
\end{gathered}
$$

More precisely, (8) is nonoscillatory if $\bar{c} \bar{r}<1 / 4$ and oscillatory if $\bar{c} \bar{r}>1 / 4$. In the limiting case $\bar{c} \bar{r}=1 / 4$, (8) is nonoscillatory if $\bar{d} \bar{r}<1 / 4$ and oscillatory if $\bar{d} \bar{r}>1 / 4$.

This result was extended in [13], where a perturbation was also allowed in the term involving derivative. More precisely, first, the differential equation

$$
\left[\left(1+\sum_{j=1}^{n} \frac{\alpha_{j}}{\log _{j}^{2}(t)}\right) x^{\prime}\right]^{\prime}+\left[\frac{1}{4 t^{2}}+\sum_{j=1}^{n} \frac{\beta_{j}}{t^{2} \log _{j}^{2}(t)}\right] x=0
$$

was considered. It was shown (Theorem 2.1 and Corollary 2.3 in [13] with suitably chosen functions $p_{0}, q_{0}, p_{1}$, and $q_{1}$ in those statements) that if there exists $k \in\{1, \ldots, n\}$, such that $\beta_{j}-\alpha_{j} / 4=1 / 4$ for $j=1, \ldots, k-1$ and $\beta_{k}-\alpha_{k} / 4 \neq 1 / 4$, then (10) is oscillatory if $\beta_{k}-\alpha_{k} / 4>1 / 4$ and nonoscillatory if $\beta-\alpha / 4<1 / 4$. If $\beta_{j}-\alpha_{j} / 4=1 / 4$ for all $j=1, \ldots, n$, then (10) is nonoscillatory. Then, using an averaging argument, it was shown in [13] that the above result remains essentially to hold when the constants $\alpha_{j}, \beta_{j}, j=1, \ldots, n$, in (10) are replaced by periodic functions. The role of constants is taken in this result by the mean values of periodic functions $\alpha_{j}(t), \beta_{j}(t)$.

As a next step, the effort was concentrated to extend the previous linear results to half-linear equations. In [12], the equation

$$
\left(r(t) \Phi\left(x^{\prime}\right)\right)^{\prime}+\frac{c(t)}{t^{p}} \Phi(x)=0
$$

with $\alpha$-periodic $r, c$, was considered. Similarly to the linear case, it was shown that (11) is oscillatory provided that $\bar{c} \bar{r}^{p-1}>$ $\gamma_{p}$ and nonoscillatory when $\bar{c} \bar{r}^{p-1}<\gamma_{p}$,

$$
\bar{r}=\frac{1}{\alpha} \int_{0}^{\alpha} r^{1-q}(t) d t, \quad \bar{c}=\frac{1}{\alpha} \int_{0}^{\alpha} c(t) d t,
$$

$q=p /(p-1)$ being the conjugate exponent of $p$; the limiting case $\bar{c} \bar{r}^{p-1}=\gamma_{p}$ remained undecided in [12]. This problem was resolved in the later paper [8]; we will mention this result later in our paper.

Here, we deal with perturbations of the Euler halflinear differential equation in full generality. We consider the following equation:

$$
\begin{aligned}
& {\left[\left(r(t)+\sum_{j=1}^{n} \frac{\alpha_{j}(t)}{\log _{j}^{2}(t)}\right)^{1-p} \Phi\left(x^{\prime}\right)\right]^{\prime}} \\
& +\left[\frac{c(t)}{t^{p}}+\sum_{j=1}^{n} \frac{\beta_{j}(t)}{t^{p} \log _{j}^{2}(t)}\right] \Phi(x)=0,
\end{aligned}
$$

with $T$-periodic functions $r, c, \alpha_{j}, \beta_{j}, r(t)>0$. One of the reasons why we consider the coefficient of $\Phi\left(x^{\prime}\right)$ in the power $1-p$ is that then this equation can be written as the first order system

$$
\begin{aligned}
& x^{\prime}=\left(r(t)+\sum_{j=1}^{n} \frac{\alpha_{j}(t)}{\log _{j}^{2}(t)}\right) \Phi^{-1}(u), \\
& u^{\prime}=-\frac{1}{t^{p}}\left(c(t)+\sum_{j=1}^{n} \frac{\beta_{j}(t)}{\log _{j}^{2}(t)}\right) \Phi(x)
\end{aligned}
$$

and perturbation terms in both equations of this system have essentially the same form.

Our main statement is based on the result of [11], where the case when $r(t) \equiv 1, c(t) \equiv \gamma_{p}$, and $\alpha_{j}, \beta_{j}$ are constants was considered. An explicit formula for the relationship between constants $\alpha_{j}$, $\beta_{j}$ which implies (non)oscillation of (13) was found. This result is recalled in the next section. In our paper, we show, similarly to the above mentioned papers, that constants $\alpha_{j}, \beta_{j}$ can be replaced by periodic functions and the resulting oscillation formula is essentially the same as that one in [11]; only constants are replaced by mean values of periodic functions appearing in perturbation terms.

\section{Preliminaries}

We start this section with a slight modification of [11, Theorem 4.1] which characterizes oscillatory properties of the following equation:

$$
\begin{aligned}
& {\left[\left(1+\sum_{j=1}^{n} \frac{\alpha_{j}}{\log _{j}^{2}(t)}\right)^{1-p} \Phi\left(x^{\prime}\right)\right]^{\prime}} \\
& +\left[\frac{\gamma_{p}}{t^{p}}+\sum_{j=1}^{n} \frac{\beta_{j}}{t^{p} \log _{j}^{2}(t)}\right] \Phi(x)=0 .
\end{aligned}
$$


Proposition 1. Suppose that there exists $k \in\{1, \ldots, n\}$ such that

$$
\begin{array}{r}
\beta_{j}+(p-1) \gamma_{p} \alpha_{j}=\mu_{p}, \quad \mu_{p}=\frac{1}{2}\left(\frac{p-1}{p}\right)^{p-1}, \\
j=1, \ldots, k-1,
\end{array}
$$

(if $k=1)$ and $\beta_{k}+(p-1) \gamma_{p} \alpha_{k} \neq \mu_{p}$. Then, (15) is oscillatory if $\beta_{k}+(p-1) \gamma_{p} \alpha_{k}>\mu_{p}$ and nonoscillatory if $\beta_{k}+(p-1) \gamma_{p} \alpha_{k}<$ $\mu_{p}$.

Note that Proposition 1 is proved in [11] for the half-linear differential equation where the term by $\Phi\left(x^{\prime}\right)$ has no power, taking the form $\left(1+\sum_{j=1}^{n} \alpha_{j} \log _{j}^{-2}(t)\right)$. However, using the following binomial expansion:

$$
\begin{aligned}
\left(1+\sum_{j=1}^{n} \frac{\alpha_{j}}{\log _{j}^{2}(t)}\right)^{1-p}= & 1+(1-p)\left(\sum_{j=1}^{n} \frac{\alpha_{j}}{\log _{j}^{2}(t)}\right) \\
& +O\left(\log ^{-4} t\right) \quad \text { as } t \longrightarrow \infty,
\end{aligned}
$$

it is not difficult to see that Theorem 4.1 of [11] can be reformulated as stated in Proposition 1.

Next, we recall the concept of half-linear trigonometric functions, see [2] or [4, Section 1.3]. Consider the following special equation of the form (2):

$$
\left(\Phi\left(x^{\prime}\right)\right)^{\prime}+(p-1) \Phi(x)=0
$$

We denote the solution of this equation satisfying the initial condition $x(0)=0, x^{\prime}(0)=1$ by $\sin _{p} t$, and its derivative $\left(\sin _{p} t\right)^{\prime}=: \cos _{p} t$. The functions $\sin _{p}, \cos _{p}$ are $2 \pi_{p}$-periodic, $\pi_{p}:=2 \pi /(p \sin (\pi / p))$, and satisfy the Pythagorean identity:

$$
\left|\sin _{p} t\right|^{p}+\left|\cos _{p} t\right|^{p}=1, \quad t \in \mathbb{R}
$$

Every solution of (18) is of the form $x(t)=C \sin _{p}(t+\varphi)$, where $C, \varphi$ are real constants; that is, it is bounded together with its derivative and periodic with the period $2 \pi_{p}$. The function $u=\Phi\left(\cos _{p} t\right)$ is a solution of the following reciprocal equation to (18):

$$
\begin{gathered}
\left(\Phi^{-1}\left(u^{\prime}\right)\right)^{\prime}+(p-1)^{q-1} \Phi^{-1}(u)=0, \\
\Phi^{-1}(u)=|u|^{q-2} u, \quad q=\frac{p}{p-1},
\end{gathered}
$$

which is an equation of the same form as (18), so the functions $u$ and $u^{\prime}$ are also bounded.

Let $x$ be a nontrivial solution of (2) and consider the modified half-linear Prüfer transformation:

$$
x(t)=\rho(t) \sin _{p} \varphi(t), \quad r^{q-1}(t) x^{\prime}(t)=\frac{\rho(t)}{t} \cos _{p} \varphi(t) .
$$

Then, the angular variable $\varphi$ satisfies the following differential equation:

$$
\begin{aligned}
\varphi^{\prime}=\frac{1}{t} & {\left[r^{1-q}(t)\left|\cos _{p} \varphi\right|^{p}\right.} \\
& \left.-\Phi\left(\cos _{p} \varphi\right) \sin _{p} \varphi+\frac{t^{p} c(t)}{p-1}\left|\sin _{p} \varphi\right|^{p}\right],
\end{aligned}
$$

see $[8]$.

The proof of our main result relies on the following averaging lemma, which can be found in [8]; see also [13, Section 5] and [15, Proposition 2].

Lemma 2. Let $\varphi$ be a solution of the following equation:

$$
\varphi^{\prime}=\frac{1}{t}\left[a(t)\left|\cos _{p} \varphi\right|^{p}-\Phi\left(\cos _{p} \varphi\right) \sin _{p} \varphi+b(t)\left|\sin _{p} \varphi\right|^{p}\right],
$$

with bounded functions $a(t)$ and $b(t), a(t)>0$, and let $T>0$. Denote the following:

$$
\theta(t):=\frac{1}{T} \int_{t}^{t+T} \varphi(s) d s .
$$

Then, $\theta$ is a solution of the following equation:

$$
\begin{aligned}
\theta^{\prime}= & \frac{1}{t}\left[A(t)\left|\cos _{p} \theta\right|^{p}-\Phi\left(\cos _{p} \theta\right) \sin _{p} \theta+B(t)\left|\sin _{p} \theta\right|^{p}\right] \\
& +O\left(\frac{1}{t^{2}}\right)
\end{aligned}
$$

with

$$
A(t)=\frac{1}{T} \int_{t}^{t+T} a(\tau) d \tau, \quad B(t)=\frac{1}{T} \int_{t}^{t+T} b(\tau) d \tau,
$$

and $\varphi(t)-\theta(t)=o(1)$ as $t \rightarrow \infty$.

The term $O\left(t^{-2}\right)$ in (25) can be written as (compare (19)) $\left(\left|\cos _{p} \theta\right|^{p}+\left|\sin _{p} \theta\right|^{p}\right) O\left(t^{-2}\right)$; hence (25) can be rewritten into the form considered later on

$$
\begin{aligned}
& \theta^{\prime}=\frac{1}{t}[(\left.A(t)+O\left(t^{-1}\right)\right)\left|\cos _{p} \theta\right|^{p}-\Phi\left(\cos _{p} \theta\right) \sin _{p} \theta \\
&\left.+\left(B(t)+O\left(t^{-1}\right)\right)\left|\sin _{p} \theta\right|^{p}\right] .
\end{aligned}
$$

\section{Main Result}

The formulation of Lemma 2 from the previous section shows why we consider the perturbations of Euler equation (1) in the form as appears in (13) and, in particular, why we consider the term by $\Phi\left(x^{\prime}\right)$ with the power $1-p$. With this power (since $(1-p)(1-q)=1)$, the function $A$ in (26) is just the mean value over the interval $[t, t+T]$ of the function $r(t)+\sum_{j=1}^{n}\left(\alpha_{j}(t) / \log _{j}^{2}(t)\right)$

Theorem 3. Let $r, c$ and $\alpha_{j}, \beta_{j}, j=1, \ldots, n$, be T-periodic continuous functions, $r(t)>0$, and denote by $\bar{r}, \bar{c}, \bar{\alpha}_{j}, \bar{\beta}_{j}, j=$ $1, \ldots, n$, their mean values over the period $T$. 
(i) If $\bar{c} \bar{r}^{p-1}>\gamma_{p}$, then (13) is oscillatory, and if $\bar{c} \bar{r}^{p-1}<\gamma_{p}$, then it is nonoscillatory.

(ii) Let $\bar{c} \bar{r}^{p-1}=\gamma_{p}$. If there exists $k \in\{1, \ldots, n\}$ such that

$$
\bar{\beta}_{j} \bar{r}^{p-1}+(p-1) \gamma_{p} \bar{\alpha}_{j} \bar{r}^{-1}=\mu_{p}, \quad j=1, \ldots, k-1,
$$

(if $k=1)$ and $\bar{\beta}_{k} \bar{r}^{p-1}+(p-1) \gamma_{p} \bar{\alpha}_{k} \bar{r}^{-1} \neq \mu_{p}$, then (13) is oscillatory if

$$
\bar{\beta}_{k} \bar{r}^{p-1}+(p-1) \gamma_{p} \bar{\alpha}_{k} \bar{r}^{-1}>\mu_{p}
$$

and nonoscillatory if

$$
\bar{\beta}_{k} \bar{r}^{p-1}+(p-1) \gamma_{p} \bar{\alpha}_{k} \bar{r}^{-1}<\mu_{p} .
$$

Proof. First of all, let us note that the statement (i) is given for completeness; it is proved in [12]. The statement (ii) for $n=1, \alpha_{1}=0$, is the main result of [8]. It remains to prove the statement (ii) in full generality.

Let $x$ be a nontrivial solution of (13) and let $\varphi$ be its Prüfer angle; that is, the solution $x$ of (13) and its quasiderivative are given by the following formulas:

$$
\begin{gathered}
x(t)=\rho(t) \sin _{p} \varphi(t) \\
\left(r(t)+\sum_{j=1}^{n} \frac{\alpha_{j}(t)}{\log _{j}^{2}(t)}\right)^{-1} x^{\prime}=\frac{\rho(t)}{t} \cos _{p} \varphi(t) .
\end{gathered}
$$

Then, $\varphi(t)=0\left(\bmod \pi_{p}\right)$ if and only if $x(t)=0$ and

$$
\begin{aligned}
\varphi^{\prime}(t) & =\frac{1}{t}\left(r(t)+\sum_{j=1}^{n} \frac{\alpha_{j}(t)}{\log _{j}^{2}(t)}\right)\left|\cos _{p} \varphi\right|^{p} \\
& =\frac{1}{t}\left(r(t)+\sum_{j=1}^{n} \frac{\alpha_{j}(t)}{\log _{j}^{2}(t)}\right)>0
\end{aligned}
$$

at these points (see (33)). Hence, (13) is oscillatory if and only if $\varphi(t)$ is unbounded as $t \rightarrow \infty$, and this happens, by Lemma 2 , if and only if its mean value over the interval $[t, t+T] \theta(t)=(1 / T) \int_{t}^{t+T} \varphi(s) d s$ is unbounded.

The function $\varphi$ is a solution of the following differential equation:

$$
\begin{gathered}
\varphi^{\prime}=\frac{1}{t}\left[\left(r(t)+\sum_{j=1}^{n} \frac{\alpha_{j}(t)}{\log _{j}^{2}(t)}\right)\left|\cos _{p} \varphi\right|^{p}-\Phi\left(\cos _{p} \varphi\right) \sin _{p} \varphi\right. \\
\left.+\frac{1}{p-1}\left(c(t)+\sum_{j=1}^{n} \frac{\beta_{j}(t)}{\log _{j}^{2}(t)}\right)\left|\sin _{p} \varphi\right|^{p}\right]
\end{gathered}
$$

that is, in differential equation (25) we have (cf. (26))

$$
\begin{gathered}
A(t)=\frac{1}{T} \int_{t}^{t+T}\left(r(s)+\sum_{j=1}^{n} \frac{\alpha_{j}(s)}{\log _{j}^{2}(s)}\right) d s, \\
B(t)=\frac{1}{(p-1) T} \int_{t}^{t+T}\left(c(s)+\sum_{j=1}^{n} \frac{\beta(s)}{\log _{j}^{2}(s)}\right) d s .
\end{gathered}
$$

Let $f$ be a continuous $T$-periodic function and $\bar{f}=(1 / T)$ $\int_{0}^{T} f(s) d s$ its mean value over the period; then integration by parts yields

$$
\begin{aligned}
\frac{1}{T} \int_{t}^{t+T} \frac{f(s)}{\log _{j}^{2}(s)} d s & \left.\frac{1}{T \log _{j}^{2}(s)} \int_{t}^{s} f(u) d u\right|_{t} ^{t+T} \\
= & \frac{1}{T} \int_{t}^{t+T}\left[\left(\frac{1}{\log _{j}^{2}(s)}\right)^{\prime} \int_{t}^{s} f(u) d u\right] d s \\
= & \frac{\bar{f}}{\log _{j}^{2}(t)}+\bar{f}\left[\frac{1}{\log _{j}^{2}(t+T)}-\frac{1}{\log _{j}^{2}(t)}\right] \\
& -\frac{1}{T} \int_{t}^{t+T}\left[\left(\frac{1}{\log _{j}^{2}(s)}\right)^{\prime} \int_{t}^{s} f(u) d u\right] d s .
\end{aligned}
$$

Since the function $f$ is bounded, there exists a constant $K>0$ such that

$$
\left|\int_{t}^{s} f(s) d s\right| \leq K, \quad \text { for } t \leq s \leq t+T
$$

and hence we can estimate the last term in the previous computation as follows:

$$
\begin{aligned}
& \left|\int_{t}^{t+T}\left[\left(\frac{1}{\log _{j}^{2}(s)}\right)^{\prime} \int_{t}^{s} f(u) d u\right] d s\right| \\
& \quad \leq K\left[\frac{1}{\log _{j}^{2}(t+T)}-\frac{1}{\log _{j}^{2}(t)}\right] \\
& \quad=\left.K T\left(\frac{1}{\log _{j}^{2}(t)}\right)^{\prime}\right|_{t=\xi}=\frac{-K T}{\xi \log \xi \log _{j}^{2}(\xi)}[1+o(1)] \\
& \quad=O\left(\frac{1}{t \log t \log _{j}^{2}(t)}\right),
\end{aligned}
$$

as $t \rightarrow \infty, \xi \in(t, T+t)$. Here, we have used that

$$
\begin{array}{r}
\left(\frac{1}{\log _{j}^{2}(t)}\right)^{\prime}=-\frac{1}{t \log t \log _{j}^{2}(t)}(1+o(1)), \\
j=1, \ldots, n,
\end{array}
$$


as can be verified by a direct computation. The same argument shows that also the term in brackets in (35) has the same asymptotic behavior as $t \rightarrow \infty$. Altogether, we have

$$
\begin{aligned}
\frac{1}{T} \int_{t}^{t+T} \frac{f(s)}{\log _{j}^{2}(s)} d s & =\frac{\bar{f}}{\log _{j}^{2}(t)}+O\left(\frac{1}{t \log t \log _{j}^{2}(t)}\right) \\
& =\frac{\bar{f}}{\log _{j}^{2}(t)}\left(1+O\left(\frac{1}{t \log t}\right)\right) .
\end{aligned}
$$

This implies that the functions $A$ and $B$ in (34) are

$$
\begin{gathered}
A(t)=\bar{r}+\left[1+O\left(t^{-1} \log ^{-1} t\right)\right] \sum_{j=1}^{n} \frac{\bar{\alpha}_{j}}{\log _{j}^{2}(t)}, \\
B(t)=\frac{1}{p-1}\left\{\bar{c}+\left[1+O\left(t^{-1} \log ^{-1} t\right)\right] \sum_{j=1}^{n} \frac{\bar{\beta}_{j}}{\log _{j}^{2}(t)}\right\} .
\end{gathered}
$$

Hence, substituting into (27), we obtain

$$
\begin{aligned}
\theta^{\prime}=\frac{1}{t}\{ & {\left[\bar{r}+\left(1+O\left(t^{-1} \log ^{-1} t\right)\right) \sum_{j=1}^{n} \frac{\bar{\alpha}_{j}}{\log _{j}^{2}(t)}+O\left(t^{-1}\right)\right] } \\
& \times\left|\cos _{p} \theta\right|^{p}-\Phi\left(\cos _{p} \theta\right) \sin _{p} \theta \\
& +\frac{1}{p-1}\left[\bar{c}+\left(1+O\left(t^{-1} \log ^{-1} t\right)\right)\right. \\
& \left.\left.\times \sum_{j=1}^{n} \frac{\bar{\beta}_{j}}{\log _{j}^{2}(t)}+O\left(t^{-1}\right)\left|\sin _{p} \theta\right|^{p}\right]\right\}
\end{aligned}
$$

Now, since all terms

$$
\frac{O(1 /(t \log t))}{\log _{j}^{2}(t)}, \quad j=1, \ldots, n, \quad O\left(t^{-1}\right) \quad \text { as } t \longrightarrow \infty
$$

are asymptotically less than $o(1) / \log _{n}^{2}(t)$, we obtain the differential equation for $\theta$ which can be written in the following form:

$$
\begin{aligned}
\theta^{\prime}=\frac{1}{t} & {\left[\left(\bar{r}+\sum_{j=1}^{n} \frac{\bar{\alpha}_{j}}{\log _{j}^{2}(t)}+\frac{o(1)}{\log _{n}^{2}(t)}\right)\left|\cos _{p} \theta\right|^{p}\right.} \\
& -\Phi\left(\cos _{p} \theta\right) \sin _{p} \theta+\frac{1}{p-1} \\
& \left.\times\left(\bar{c}+\sum_{j=1}^{n} \frac{\bar{\beta}_{j}}{\log _{j}^{2}(t)}+\frac{o(1)}{\log _{n}^{2}(t)}\right)\left|\sin _{p} \theta\right|^{p}\right] .
\end{aligned}
$$

This equation is a "Prüfer angle" equation for the following second-order half-linear differential equation:

$$
\begin{aligned}
& {\left[\left(\bar{r}+\sum_{j=1}^{n} \frac{\bar{\alpha}_{j}}{\log _{j}^{2}(t)}+\frac{o(1)}{\log _{n}^{2}(t)}\right)^{1-p} \Phi\left(x^{\prime}\right)\right]^{\prime}} \\
& \quad+\frac{1}{t^{p}}\left(\bar{c}+\sum_{j=1}^{n} \frac{\bar{\beta}_{j}}{\log _{j}^{2}(t)}+\frac{o(1)}{\log _{n}^{2}(t)}\right) \Phi(x)=0,
\end{aligned}
$$

which is the same as the following equation:

$$
\begin{aligned}
& {\left[\left(1+\sum_{j=1}^{n} \frac{\bar{\alpha}_{j} / \bar{r}}{\log _{j}^{2}(t)}+\frac{o(1)}{\log _{n}^{2}(t)}\right)^{1-p} \Phi\left(x^{\prime}\right)\right]^{\prime}} \\
& \quad+\frac{1}{t^{p}}\left(\bar{c} \bar{r}^{p-1}+\sum_{j=1}^{n} \frac{\bar{\beta} \bar{r}^{p-1}}{\log _{j}^{2}(t)}+\frac{o(1)}{\log _{n}^{2}(t)}\right) \Phi(x)=0 .
\end{aligned}
$$

Suppose that assumptions (ii) of Theorem 3 are satisfied and that (29) holds for $k \in\{1, \ldots, n-1\}$. Then, (45) is oscillatory as a direct consequence of Proposition 1. If (29) holds for $k=n$, let $\varepsilon>0$ be so small that still

$$
\bar{r}^{p-1} \bar{\beta}_{n}-\varepsilon+(p-1) \gamma_{p}\left(\bar{r}^{-1} \bar{\alpha}_{n}-\varepsilon\right)>\mu_{p}
$$

and consider the following equation:

$$
\begin{aligned}
& {\left[\left(1+\sum_{j=1}^{n-1} \frac{\bar{\alpha}_{j} / \bar{r}}{\log _{j}^{2}(t)}+\frac{\bar{\alpha}_{n} / \bar{r}-\varepsilon}{\log _{n}^{2}(t)}\right)^{1-p} \Phi\left(x^{\prime}\right)\right]^{\prime}} \\
& \quad+\frac{1}{t^{p}}\left(\bar{c} \bar{r}^{p-1}+\sum_{j=1}^{n-1} \frac{\bar{\beta}_{j} \bar{r}^{p-1}}{\log _{j}^{2}(t)}+\frac{\bar{\beta}_{n} \bar{r}^{p-1}-\varepsilon}{\log _{j}^{2}(t)}\right) \Phi(x)=0 .
\end{aligned}
$$

This equation is a Sturmian minorant for $t$ sufficiently large (when the $o(1)$ term in (45) is less than $\varepsilon$ ) of (45) and (46) implies by Proposition 1 that this minorant equation is oscillatory, and hence (45) (which is the same as (44)) is oscillatory as well. This means that the Prüfer angle $\theta$ of a solution of (44) is unbounded, and by Lemma 2 the Prüfer angle $\varphi$ of a solution of (13) is unbounded as well. This means that (13) is oscillatory. A slightly modified argument implies that (13) is nonoscillatory provided that (30) holds.

\section{Remarks and Comments}

(i) In Lemma 2, the constant $T$ is any positive real constant. In the original version of this Lemma in [15], this number is the period of periodic functions in (25). As observed in [13], the statement of lemma 1 can be extended so that it applies to equations with bounded coefficients; see [13, Section 5] for details. This opens a space for a more general treatment of perturbations of the Euler type differential equation when perturbation functions are of larger class than periodic ones. A first step along this line has been made in [16] where 
a half-linear differential equation with asymptotically almost periodic coefficients is investigated.

(ii) In [17], a power comparison theorem (with respect to the power $p$ in the function $\Phi(x)=|x|^{p-2} x$ ) for perturbed Euler type differential equation is established. This result is extended to the Riemann-Weber half-linear differential equation in [18]. A natural research problem is to deal with power comparison theory for general perturbations suggested by (13).

(iii) Another research problem is associated with the concept of Karamata regularly varying functions; see [19-23]. In these papers, half-linear equations with coefficients which are regularly varying functions are considered, and it is shown that then solutions of these equations also behave regularly, in a certain sense. Since the idea of the proofs of results of those papers consists in comparing the investigated equation with the Euler or Riemann-Weber equation, a natural idea is to investigate asymptotic properties of (15) with regularly varying functions $r, c, \alpha_{j}$, and $\beta_{j}$.

\section{Acknowledgments}

This research is supported by Grant 201/11/0768 of the Grant Agency of the Czech Republic and the Research Project MUNI/A/0964/2009 of Masaryk University.

\section{References}

[1] Á. Elbert, "Asymptotic behaviour of autonomous half-linear differential systems on the plane," Studia Scientiarum Mathematicarum Hungarica, vol. 19, no. 2-4, pp. 447-464, 1984.

[2] Á. Elbert, "A half-linear second order differential equation," in Qualitative Theory of Differential Equations, Vol. I, II, vol. 30 of Colloquia Mathematica Societatis János Bolyai, pp. 153-180, North-Holland, Amsterdam, The Netherlands, 1981.

[3] T. Kusano, Y. Naito, and A. Ogata, "Strong oscillation and nonoscillation of quasilinear differential equations of second order," Differential Equations and Dynamical Systems, vol. 2, no. 1, pp. 1-10, 1994.

[4] O. Došlý and P. Řehák, Half-Linear Differential Equations, vol. 202 of North-Holland Mathematics Studies, Elsevier, Amsterdam, The Netherlands, 2005.

[5] Á. Elbert and A. Schneider, "Perturbations of the half-linear Euler differential equation," Results in Mathematics, vol. 37, no. 1-2, pp. 56-83, 2000.

[6] O. Došlý, "Perturbations of the half-linear Euler-Weber type differential equation," Journal of Mathematical Analysis and Applications, vol. 323, no. 1, pp. 426-440, 2006.

[7] O. Došlý and H. Haladová, "Half-linear Euler differential equations in the critical case," Tatra Mountains Mathematical Publications, vol. 48, pp. 41-49, 2011.

[8] O. Došlý and P. Hasil, "Critical oscillation constant for halflinear differential equations with periodic coefficients," Annali di Matematica Pura ed Applicata, vol. 190, no. 3, pp. 395-408, 2011.

[9] O. Došlý and S. Fišnarová, "Half-linear oscillation criteria: perturbation in term involving derivative," Nonlinear Analysis: Theory, Methods \& Applications, vol. 73, no. 12, pp. 3756-3766, 2010.
[10] O. Došlý and S. Fišnarová, "Two-parametric conditionally oscillatory half-linear differential equations," Abstract and Applied Analysis, vol. 2011, Article ID 182827, 16 pages, 2011.

[11] O. Došlý and H. Funková, "Perturbations of half-linear Euler differential equation and transformations of modified Riccati equation," Abstract and Applied Analysis, vol. 2012, Article ID 738472, 19 pages, 2012.

[12] P. Hasil, "Conditional oscillation of half-linear differential equations with periodic coefficients," Archivum Mathematicum, vol. 44, no. 2, pp. 119-131, 2008.

[13] H. Krüger and G. Teschl, "Effective Prüfer angles and relative oscillation criteria," Journal of Differential Equations, vol. 245, no. 12, pp. 3823-3848, 2008.

[14] K. M. Schmidt, "Oscillation of the perturbed Hill equation and the lower spectrum of radially periodic Schrödinger operators in the plane," Proceedings of the American Mathematical Society, vol. 127, no. 8, pp. 2367-2374, 1999.

[15] K. M. Schmidt, "Critical coupling constants and eigenvalue asymptotics of perturbed periodic Sturm-Liouville operators," Communications in Mathematical Physics, vol. 211, no. 2, pp. 465-485, 2000.

[16] P. Hasil and M. Veselý, "Oscillation of half-linear differential equations with asymptotically almost periodic coefficients," Advances in Difference Equations, vol. 2013, article 122, 2013.

[17] J. Sugie and N. Yamaoka, "Comparison theorems for oscillation of second-order half-linear differential equations," Acta Mathematica Hungarica, vol. 111, no. 1-2, pp. 165-179, 2006.

[18] G. Bognár and O. Došlý, "A remark on power comparison theorem for half-linear differential equations," Mathematica Bohemica, vol. 133, no. 2, pp. 187-195, 2008.

[19] J. Jaroš, K. Takaŝi, and T. Tanigawa, "Nonoscillation theory for second order half-linear differential equations in the framework of regular variation," Results in Mathematics, vol. 43, no. 1-2, pp. 129-149, 2003.

[20] J. Jaroš, K. Takaŝi, and T. Tanigawa, "Nonoscillatory half-linear differential equations and generalized Karamata functions," Nonlinear Analysis: Theory, Methods \& Applications, vol. 64, no. 4, pp. 762-787, 2006.

[21] T. Kusano, J. Manojlović, and T. Tanigawa, "Existence of regularly varying solutions with nonzero indices of half-linear differential equations with retarded arguments," Computers \& Mathematics with Applications, vol. 59, no. 1, pp. 411-425, 2010.

[22] Z. Pátíková, "Asymptotic formulas for non-oscillatory solutions of perturbed half-linear Euler equation," Nonlinear Analysis: Theory, Methods \& Applications, vol. 69, no. 10, pp. 3281-3290, 2008.

[23] Z. Pátíková, "Asymptotic formulas for nonoscillatory solutions of conditionally oscillatory half-linear equations," Mathematica Slovaca, vol. 60, no. 2, pp. 223-236, 2010. 


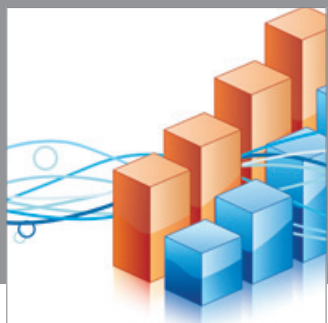

Advances in

Operations Research

mansans

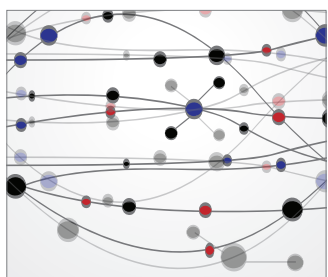

The Scientific World Journal
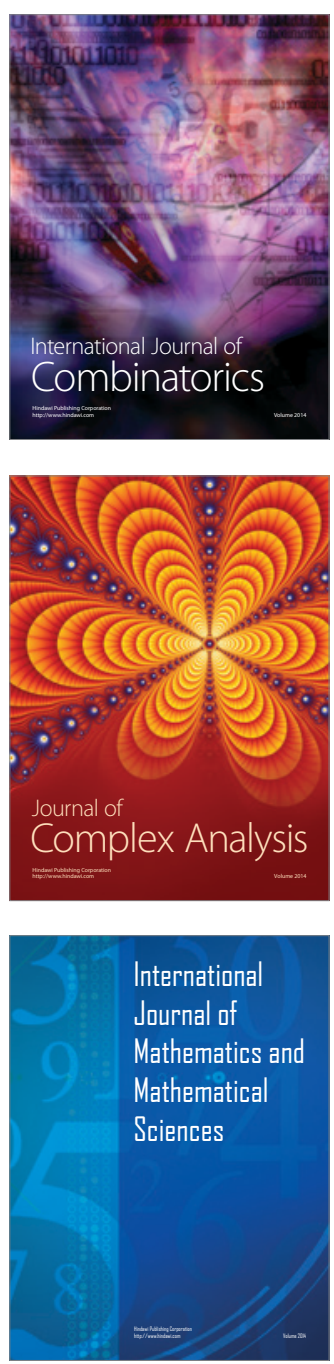
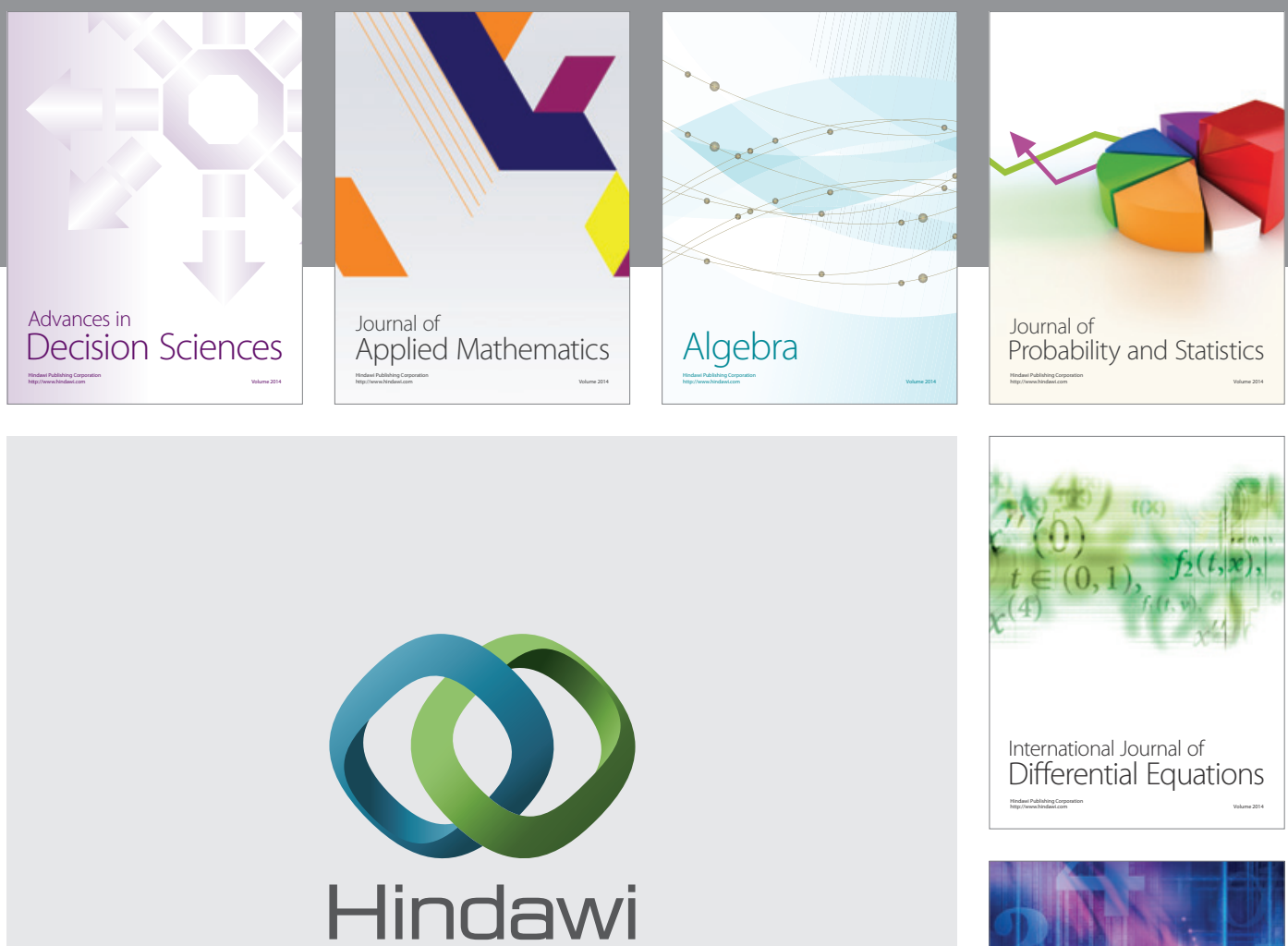

Submit your manuscripts at http://www.hindawi.com
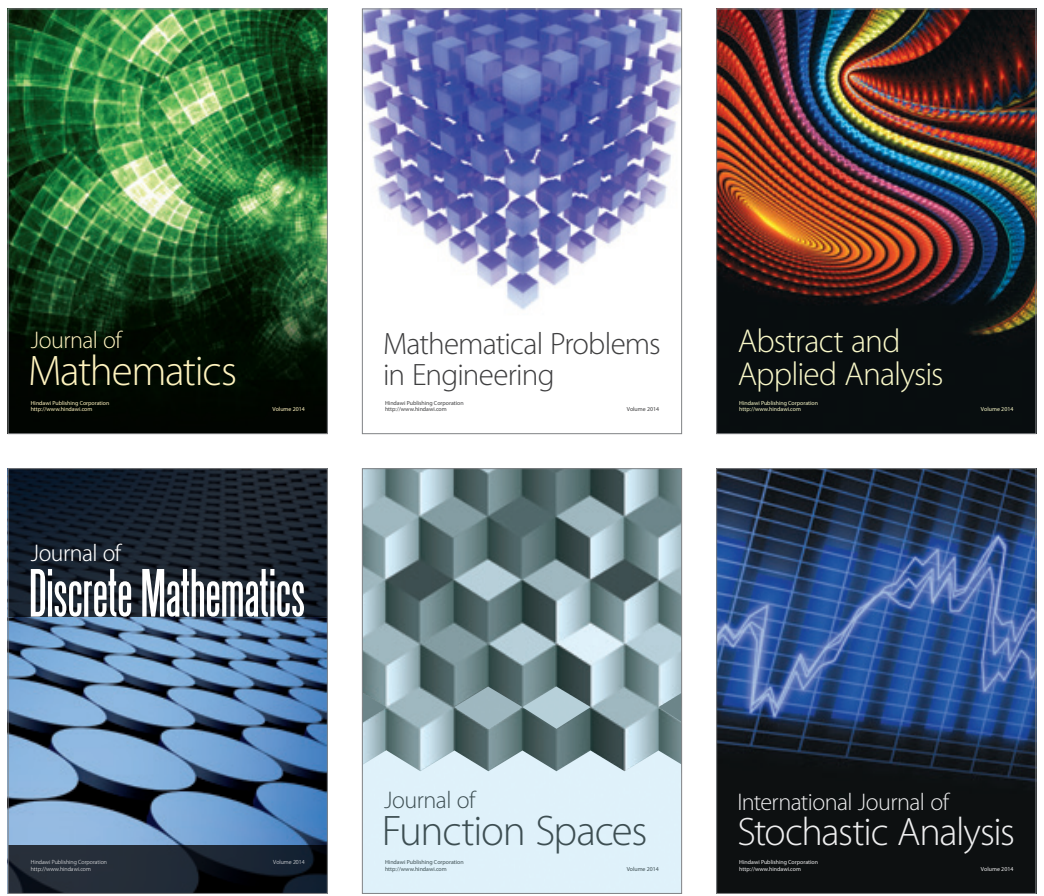

Journal of

Function Spaces

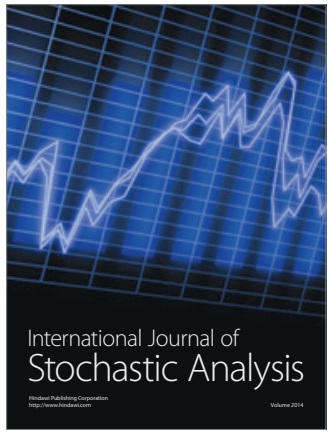

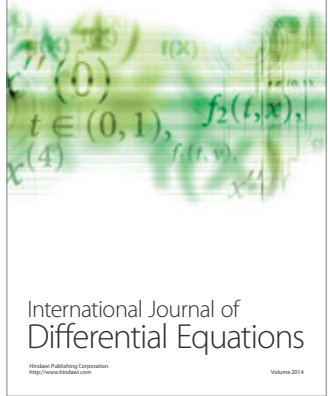
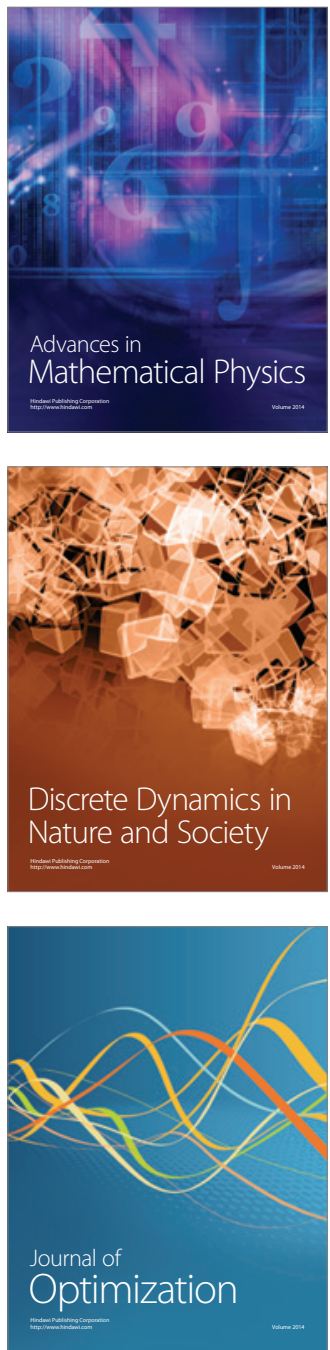